\title{
About solving linear integer programs through Hermite Normal Form decomposition
}

\author{
J.MAUBLANC, A.QUILLIOT \\ Université BLAISE PASCAL, \\ ISIMA, BP 125, Campus des cézeaux, 63173 CLERMONT-FD, FRANCE, Tel 73 \\ 405004, Fax 73405001
}

\begin{abstract}
This paper discusses the ways Hermite Normal Form decompositions may be used in order to solve integer linear programs.
\end{abstract}

Keywords

Hermite Normal Form, Integer Linear Programming

\section{INTRODUCTION}

Integer linear programming (ILP) is a central combinatorial optimization problem. More than the boolean satisfiability problem 3-SAT, which is used as the reference problem in complexity theory (Garey and Johnsson, 1979), it provides a simple way of expressing problems belonging to the class NP-Time. In many cases, this translation process can be automatized. Thus, ILP plays the role of some kind of universal problem inside NP-Time, in a more practical way than the other NP-Complete problems.

The ILP (or MLP: Mixed linear programming) formalism is mostly applied to (Schrijver, 1986) combinatorial problems involving decision variables, planning and scheduling problems, and diagnostic problems for fault localization. Its universal status provides a justification to the very huge amount of work which has been developped about it, as well as the large variety of software built in order to handle it (CPLEX, XMP, OSL, ...). Recently, research concerning constraint programming languages (CHIP, CLP, PECOS, PROLOG III) have focused on integrating efficient ILP libraries inside logic programming formalisms.

The most classical methods for dealing with ILP are derivations of the Simplex algorithm. Some stem from specificities of the underlying problem (total unimodularity, facet generation). Others are designed for general purposes and involve cutting plane generation mechanisms (Gomory, 1963; Glover, 1966; Chvatal, 1985), branch and bound combined with some kind of relaxation, or Benders decomposition (Dakin, 1965; Schrijver, 1986; Srinivasan, 1965).

Something somewhat disappointing is that few among these methods can exploit the geometrical properties of the domains they consider or the arithmetical properties of the integral numbers which describe the problem. While Lenstra result about the polynomiality of ILP in fixed dimension is based upon considerations about the thickness of a polyedron, it doesn't yield any efficient algorithm. In the same way, Hermite and Smith decomposition processes applied to the constraint matrix allow to solve linear diophantine equation systems in a polynomial time, but are scarcely used to solve more general ILP problems.

For this reason, we investigate here what kind of geometrical or arithmetical arguments 
may be introduced in order to help in dealing with ILP.

First, we notice that if a given square matrix defines a fractional vertex of some polyedron, then setting this matrix in Hermite Normal Form means performing some change of variables which preserves the integrality constraint, provides some local regularization of the polyedron in the neighbourhood of this vertex, and makes appear an integral "candidate" vector. It induces the possible implementation of cutting plane methods and of branch and bound methods.

Next we combine these methods with a stochastic control allowing the current vertex to jump throughout the polyedron, in search of the best areas for cutting it.

We end by discussing the impact of the thickness of the constraint polyedron on the behavior of descent algorithms for ILP, and tried to provide methods for increasing this thickness through well-choosen variable changes.

\section{HERMITE NORMAL FORM AND DIOPHANTINE SYSTEMS}

\section{Recall: Normal Hermite Form.}

A $n^{*} m(n \geq m)$ integral matrix B is said to be in Hermite Normal Form (HNF), if it is lower triangular on its $m$ first columns, null on its other columns, if all the $B_{i, i}, i=1 . . m$, are positive and if on any row $\mathrm{i}=1 \mathrm{..m}$, its non diagonal elements are negative or null and with absolute value strictly less than the corresponding $\mathrm{B}_{\mathrm{i}, \mathrm{i}}$.

It is known (Schrijver, 1986) that for any integral matrix $n^{*} m$ matrix $C$, with range $m \leq$ $\mathrm{n}$, there exists an integral $n * n$ matrix $U$, with determinant equal to 1 or -1 , such that $B=C U$ be in HNF. Then (Damich, Kannan and Trotter, 1987), B is unique and computing U and B can be done in polynomial time through modulo determinant arithmetic. Practically, one needs to be sure that the largest common divisor of all the $n * n$ subdeterminants of $A$ is not too large in order to avoid handling very large integers while performing this process.

It follows that a linear diophantine equalities system $\mathrm{Cx}=\mathrm{b}, \mathrm{x}$ in $\mathrm{Z}^{\mathrm{n}}$ (b integral with length $\mathrm{m}, \mathrm{C}$ as above) can be solved in polynomial time (Schrijver, 1986), through the change of variable $\mathrm{x}=\mathrm{UX}$, which preserves the integrality constraint.

Let us consider now the following satisfiability problem:

Problem (P): $\left\{\right.$ Find $x$ in $Z^{n}$, such that $A x \leq b$, where $A$ is an integral $n^{*} m$ matrix with rank $n$ and $b$ is an integral vector with length $m \geq n$; ;

and let us denote by $\mathrm{P}^{*}$ the polyedron defined by: $\mathrm{P}^{*}=\left\{\mathrm{x}\right.$ in $\mathrm{Q}^{\mathrm{n}}$ such that $\left.\mathrm{Ax} \leq \mathrm{b}\right\}$.

Let us suppose that $I$ included into $\{1 . . \mathrm{m}\}$ defines a vertex of $\mathrm{P}^{*}$ : that means that $I$ is such that the submatrix $\mathrm{A}^{\mathrm{I}}$ induced by the rows of $\mathrm{A}$ which are indexed on $\mathrm{I}$ is inversible and satisfies

$$
\text { A. }\left(\mathrm{A}^{\mathrm{I}}\right)^{-1} \cdot \mathrm{b}^{\mathrm{I}} \leq \mathrm{b} \text {. }
$$

We may apply to $P$ the following change of variable:

$\mathrm{x}=\mathrm{UX}, \mathrm{U}$ being an integral matrix with determinant 1 or -1 such that $\mathrm{A}^{\mathrm{I}} . \mathrm{U}$ is in HNF.

This change of variable has several consequences:

- It induces a local regularization of the polyedron $\mathrm{P}^{*}$ at the neighbourhood of the vertex defined by $\mathrm{I}$, that means it increases the conical angulus defined by $\mathrm{I}$, making more efficient descent methods initialized on this vertex.

- It yields cutting planes, called Hermite Cutting Planes associated with I, and some peculiar integral vector $\beta(I)$, which we call "Candidate vector associated with $I$ ". We only have to set $\mathrm{B}=\mathrm{AU}$ and to label the row indices of $\mathrm{B}$ in such a way that $\mathrm{I}=1 . . \mathrm{n}$, and to write:

$\beta_{1}=\left[b_{1} / B_{1,1}\right]=$ Integral lower bound of $b_{1} / B_{1} 1,1$;

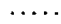


$\beta_{n}=\left[\left(b_{n}-B_{n, 1} \cdot \beta_{1}-B_{n, n-1} \cdot \beta_{n-1}\right) / B_{n, n}\right]$

and deduce the following constraints on : $\mathrm{X}_{\mathrm{i}} \leq \beta_{\mathrm{i}}$, i dans $1 . . \mathrm{n}$, as well as an integral vector $\beta(\mathrm{I})=\left(\beta_{1} \ldots \beta_{\mathrm{n}}\right)$.

These constraints are called "Hermite cuts associated with I" and the vector $\beta(\mathrm{I})$ is called "Candidate vector associated with I". One check that these Hermite cuts are not Gomory cuts.

\section{AN ALGORITHM BASED ON HERMITE CUTS}

From the above remarks, we deduce the following algorithm:

HERMI1

Input: the system $A x \leq b ; x$ in $Z^{n}$;

Output: a solution of this system if there exists some;

(Not Stop; While Not Stop do

\{Determine I included into $\{1 . . \mathrm{m}\}$, which defines a vertex of $\mathrm{P}^{*}$ and which is minimal for the lexicographic order; $(*)$

If I doesn't exist then Stop (Failure) else

\{Perform from I the Hermite variable change, compute the Hermite cuts, insert them inside the definition of $P$, at the end of the array $A$;

Let $\alpha$ be the first element in I; For $\mathrm{i}<\alpha$, replace $b_{i}$ by $b_{i}-1 ;\left(^{* *}\right)$

Compute the Candidate vector $\beta(\mathrm{I})$ associated with $\mathrm{I}$; If $\beta(\mathrm{I})$ is solution of $(\mathrm{P})$ then Stop (Success) \} \}]

\section{Remark I}

Making reference to the lexicographic order in the instructions $\left(^{*}\right)$ and $\left(^{* *}\right)$ strengthens the system with what we call Lexicographical Cuts. In fact, for $i<\alpha$ above, it will not be possible to find any integral vector $x$ (writen in relation with the current basis), such that $A^{i} \cdot x \geq b_{i}$.

\section{Remark 2}

Using the candidate vector $\beta(\mathrm{I})$ speeds the convergence of the method, since it is not anymore necessary to wait (as it was the case for the Gomory cut mechanism) for the vertex associated with I to be integral. It avoids the utilisation of fractionnal number during the execution of the algorithm.

\section{Remark 3}

In order to make HERMIl efficient, we need to keep the system under the form of a family of diophantine linear inequalities. For this, we work through the dual version of the Simplex algorithm on the dual version of the program which defines $\mathrm{P}^{*}$. In order to avoid the appearance of very large numbers during the execution of HERMI1, we compute the HNF of a given square matrix through an algorithm (Damich, Kannan and Trotter, 1987) which uses modulo determinant arithmetic.

\section{Theorem 1}

If $\mathrm{P}^{*}$ is bounded, then HERMI1 solves $(\mathrm{P})$ in a finite number of iterations.

\section{Proof}

We proceed here the same way as in the proof of the convergence of the Gomory cuts algorithm. Let us suppose that some execution of HERMI1 doesn't end and proceed by induction on $\mathrm{n}$. At some time during the process, the value of the index $\alpha$ used in the 
description of HERMI 1 becomes constant and equal to 1 . If it were false, the first coordinate of $\mathrm{b}$ would indefinitely decrease (because of the instruction $\left(^{*}\right)$ ) and the polyedron $\mathrm{P}^{*}$ wouldn't be bounded. Thus, from some time during the process, the first constraint in (P) remains writen in an unmodified way: $A_{\alpha, 1} X_{1} \leq b_{\alpha}, b_{\alpha}$ being divisible by $A_{\alpha, 1}$.

From this time, a short reasoning makes appear that the process behaves as if it were working only on the variables $X_{2}, \ldots, X_{n}$, with $X_{1}$ remaining constrained to be equal to $b_{\alpha} / A_{\alpha, 1}$. Then it becomes possible to conclude by induction. END.

\section{Introduction of a stochastic control}

HERMI1 may converge relatively slowly, as it is the case for the general Gomory algorithm. In such a situation, the vertex associated with $I$ is almost not affected by the introduction of the new cuts, and the candidate vector $\beta(I)$ remains far from $P^{*}$. Its is possible to counter this drawback by using a linear control, whose parameters are modified in a stochastic way, and which makes the current vertex associated with I jump throughout the polyedron $\mathrm{P}^{*}$. This very simple trick prove itself to improve the algorithm in a surprinsing way.

HERMI 1 can be then rewriten as an algorithm HERMI2 by replacing the $(*)$ instruction of HERMIl with the following instruction (*bis) and by suppressing the instruction (**):

Instruction (*bis):

Randomly generate a vector $\mathrm{c}$ in $\mathrm{Z}^{\mathrm{n}}$;

Determine $I$ included into $\{1 . . \mathrm{m}\}$ and defining a vertex of $\mathrm{P}^{*}$ which maximizes c.x;

\section{A TRY AND TEST ALGORITHM BASED ON HERMITE CUTS}

An other way to take profit of the Hermite variable change techniques consists in performing a try/test tree search pruned by the insertion of the Hermite cuts mechanism and by a success test on the candidate vector. in order to describe it, let us consider the following problem (P):

( Find $\mathrm{x}$ in $\mathrm{Z}^{\mathrm{n}}$ such that $\mathrm{Ax} \leq \mathrm{b}$, A being a $\mathrm{n}^{*} \mathrm{~m}$ integral matrix with rank $\mathrm{n}, \mathrm{b}$ being an integral vector with length $\mathrm{m}$ \};

The change of variable $x=U X$ associated with the FNH decomposition of the submatrix $A^{I}$ defined by some vertex of the polyedron $P^{*}$ makes appear a first Hermite cut $X_{1} \leq \beta_{1}$ (cf previous notations). Then this cut yields in a natural way a binary branching process, defined by the two following options:

$$
X_{1}=\beta_{1} \text { and } X_{1} \leq \beta_{1}-1 \text {; }
$$

The first one will be tried first, as the most promizing and since it decreases the dimension of the problem.

This branching process will be summarized as follows:

\section{BRANCH-HERMI}

(1). Randomly generate $\mathrm{c}$ in $\mathrm{Z}^{\mathrm{n}}$; $\left(^{*}\right)$

Solve the program: $\left\{A y \leq b, y\right.$ in $Q^{n}$, zmin $\left.=c . y\right\}$;

If this program admits no solution then Failure and Stop

else continue after denoting by I the subset $\{1 \ldots \mathrm{m}\}$ which defines a vertex of the polyedron $\left\{\mathrm{Ax} \leq \mathrm{b}, \mathrm{y}\right.$ in $\mathrm{Q}^{\mathrm{n}}$ \} which is a solution of this program;

(2). Perform the change of variable $\mathrm{x}=\mathrm{UX}$ associated with the FNH decomosition of $\mathrm{A}^{\mathrm{I}}$;

On the basis of this reformulation of the system, generate the Hermite cuts $X_{i} \leq \beta_{i}, i=$ 
1..n, and the candidate vertex $\beta(\mathrm{I})$ associated with $\mathrm{I}$ and defined by : $\beta(\mathrm{I})=\beta_{1} . . \beta_{\mathrm{n}}$; If $A . \beta \leq b$ then Success and Stop else continue;

(3). Recursively solve the system (P1), obtained from (P) after reformulation through the change of variable $x=U X$ and insertion of the Hermite cuts $X_{i} \leq \beta_{i}, i=2 . n$, and of the constraint $X_{1}=\beta_{1}$;

If some solution appears, then Success, else recursively solve the system (P2) obtained from $(P)$ after reformulation through the change of variable $\mathrm{x}=\mathrm{UX}$ and insertion of the cuts $X_{i} \leq \beta_{i}, i=2 . . n$ and of the constraint $X_{1} \leq \beta_{1}-1$.

\section{Explanations}

The above process is doubly filtered: on one side, the test on the integral relaxation of the program (P) allows an anticipation on failure and on an other side, the test on the candidate vertex helps in detecting the success situations. It also helps in handling rounding: in fact, the main part of the above algorithm may be writen while using only integers.

\section{NUMERICAL TESTS AND EXTENSIONS}

We tested the above methods by randomly generating linear systems $\mathrm{Ax} \leq \mathrm{b}$ which admits at least one solution and by adding them some constraint $\mathrm{cx} \leq \mathrm{k}+1$, $\mathrm{k}$ being the integral upper bound of the minimal fractional value of $\mathrm{cx}$, taken for all the vectors $\mathrm{x}$ such that $\mathrm{Ax} \leq \mathrm{b}$.Thus, the systems generated this way were "semantic free", which means without any underlying structure.

For every example generated this way, we tested both HERMI1 and HERMI2, while focalizing our attention on the number executions of the main loop and comparing with the behaviour of the classical Gomory algorithm (Gomory, 1963).

We also tested BRANCH-HERMI, as well as its deterministic version DET-BRANCHHERMI (obtained after replacing the first instruction $\left(^{*}\right)$ by an instruction " $c:=0$ ") while counting the number of nodes of the search tree which are visited during the execution of the process, and comparing with the behaviour of a simple branch and bound scheme RELAXSIMPLE, combined a simple integral relaxation (Dakin, 1965; Srinivasan, 1965).

These experiments, realized for examples which involve from 10 to 40 variables and between 20 and 100 constraints, make appear:

- an iteration number almost 10 times less for HERMI2 than for HERMI1, and almost 3 times less for HERMI1 than for GOMORY.

- a node number almost 3 times less for BRANCH-HERMI than for RELAX-SIMPLE.

Still, one must take into account, while evaluating these results, the additionnal costs due to the FNH decomposition processes involved in the execution of HERMI1, HERMI2 and BRANCH-HERMI and the global tendancy of the method to generate very large numbers.

Comparizon between GOMORY, HERMII and HERMI2

\begin{tabular}{llll} 
& GOMORY & HERMI1 & HERMI2 \\
\hline 10 & 27 & 11 & 3.4 \\
\hline 20 & 52 & 26 & 8.2 \\
\hline 30 & 97 & 38 & 16 \\
\hline 40 & 201 & 80 & 32 \\
\hline
\end{tabular}


Comparizon between SIMPLE-RELAX, BRANCH-HERMI, DET-BRANCH-HERMI

\begin{tabular}{llcc} 
& SIMPLE-RELAX & BRANCH-HERMI & DET-BRANCH- \\
\hline HERMI & & & \\
\hline 10 & 13 & 3.5 & 5.2 \\
\hline 20 & 32 & 8 & 13 \\
\hline 30 & 58 & 17 & 25 \\
\hline 40 & 105 & 33 & 45 \\
\hline
\end{tabular}

\section{MORE ON GEOMETRY: THICKNESS OF A POLYEDRON AND RELATED VARIABLE CHANGES}

We just saw the way a Hermite variable change may help in generating cuts or pruning a tree search in order to solve an ILP. We are going to see now that other kind of improvement may come from some well choosen change of variable.

Its is known (LENSTRA, 1983) that if a $\mathrm{n}$ dimensional polyedron $\mathrm{P}$ is bounded and doesn't contain any integral vector, then there exists an integral non null vector $\mathrm{c}$ such that for any pair $\mathrm{x}, \mathrm{x}^{\prime}$ of vectors in $\mathrm{P}$ :

$$
\text { c. }\left(x-x^{\prime}\right) \leq n(n+1) \cdot 2^{1 / 2} \cdot n \cdot(n+1)+1 \text {. }
$$

This result yields the famous Lenstra Theorem about the complexity of ILP in fixed dimension.

We define here, what we call the thickness $T h(P)$ of $P$ :

$$
\operatorname{Th}(\mathrm{P})=\underset{\mathrm{Inf}}{\operatorname{Inf}} \quad \begin{aligned}
& \text { Sup c. }\left(\mathrm{x}-\mathrm{x}^{\prime}\right) . \\
& \mathrm{c} \text { dans } \mathrm{R}^{\mathrm{n}} /\|\mathrm{c}\|=1 \quad \mathrm{x}^{\prime} \text { dans } \mathrm{P}
\end{aligned}
$$

Intuitively, we feel that this quantity $\mathrm{Th}(\mathrm{P})$ must be related with the probability of existence of an integral vector in P. As a matter of fact, we may assert:

\section{Theorem 2}

If $T h(P) \geq 2 n$, then $P$ contains at least one integral vector.

\section{Proof}

Let us first prove that if $T h(P) \geq 2 n$ and if $u_{1} . . u_{n}$ are vectors in $R^{n}$ which define an euclidian basis of $R^{n}$, then for any $i$ in $1 . . n$, there exists $x_{i}$ in $P$ such that $x_{i}+2 n$. $_{i}$ is also in $\mathrm{P}$. We clearly may suppose that $\mathrm{P}$ is bounded.

For any $\mathrm{u}$ such that $\|\mathrm{u}\|=1$, we may set:

$$
\mathrm{k}_{\mathrm{u}}=\underset{\mathrm{x} \text { in } \mathrm{C}}{\operatorname{Suph}} \lambda \text { such that } \mathrm{x}+\lambda \text {.u is in } \mathrm{C} \text {; }
$$

For such an $u$, let us separate the product $P * P$ and $Q_{u}=\left\{x, y\right.$ in $\left.R^{2 n} / y-x=k_{u} \cdot u\right\}$ with a hyperplan $a . x+b . y=c$ such that for any $x, y$ in $Q u: a . x+b . y \geq c$. (E1)

From (E1) it comes that $b=-a$ and $c=k_{u} b$.u. By choosing $x_{0}$ in $P$ such that :

$$
\text { a. } x_{0}=\gamma_{0}=\text { Sup a. } x, x \text { in } P \text {, }
$$

we see that for any $y$ in P: a.y $\leq \gamma_{o}$ and a.y $\geq \gamma_{0}-k_{u} \cdot b . u$;

If we suppose $\|\mathrm{a}\|=1$, then : $\mathrm{k}_{\mathrm{u}}$.b.u $\leq \mathrm{k}_{\mathrm{u}} \cdot\|\mathrm{a}\|$. $\|\mathrm{u}\| \leq \mathrm{k}_{\mathrm{u}}$; 
which means, because of (E2), that $T h(P) \leq k_{u}$.

Then we only have to compute $k_{u}$ for any $u:=u_{i}, i=1 . . n$, in order to get our initial assertion.

Let us suppose now that $x_{1} . . x_{n}$ exist in $P$ such that for any $i$ in $1 . . n, x_{i}^{\prime}=x_{i}+2 n . u_{i}$ is also in $P, u_{1} . . u_{n}$ defining an euclidian basis of $R^{n}$. We may set:

- for any $i$ in $1 . . n, x_{i}=n . a_{i}+p_{i}$ with $a_{i}$ in $Z^{n}$ and $0 \leq p_{i}<n .1$;

and also :

$-\mathrm{x}=$ gravity center of the $\mathrm{x}_{\mathrm{i}}, \mathrm{x}_{\mathrm{i}} \mathrm{i}, \mathrm{i}$ in $1 . . \mathrm{n}=\mathrm{A}+\mathrm{C} / \mathrm{n}+\mathrm{U}$,

with $A=\Sigma a_{i}, \quad C=\Sigma p_{i}, \quad U=\Sigma u_{i} \quad$;

$-\mathrm{x}^{*}=\mathrm{A}+\mathrm{U}$.

$\mathrm{i}$ in $1 . . \mathrm{n} \quad \mathrm{i}$ in $1 . . \mathrm{n} \quad \mathrm{i}$ in $1 . . \mathrm{n}$

If $x^{*}$ is in $P$ then it is over, else there exists $\lambda$ in $R^{n}, \mu$ in $R$, such that: (Séparation)

$\Sigma\left|\lambda_{i}\right|=1 ; \quad \lambda . x^{*}<\mu$; for any $i$ in $1 . . n, \lambda . x_{i}$ and $\lambda . x^{\prime} i \geq \mu$.

$\mathrm{i}$ in $1 . . \mathrm{n}$

Let us then set: $I=\left\{i\right.$ in $\left.1 . . n / \lambda_{i} \geq 0\right\}, J=\{1 . . n\}-I$, and also, for any $i$ in $1 . . n$ :

$y_{i}=x_{i}$ if $i$ in $I$ and $y_{i}=x_{i}^{\prime}$ else ;

$y^{\prime} i=x^{\prime} i$ if $i$ in $I$ and $y^{\prime} i=x_{i}$ else .

In any case, we may write: $y_{i}=n \cdot b_{i}+p_{i}$ with $b_{i}$ in $Z^{n}$;

$y_{i}^{\prime}=y_{i}+2 n \cdot v_{i}$ (with $v_{i}=u_{i}$ if $i$ in $I$ and $v_{i}=-u_{i}$ else);

Then $x^{*}$ may be rewriten $B+V$ (with $B=\Sigma b_{i}$ and $V=\Sigma v_{i}$ )

$\mathrm{i}$ in $1 . . n \quad \mathrm{i}$ in $1 . . n$

while $\lambda$ and $\mu$ satisfy:

for any $\mathrm{i}$ in 1..n, $\lambda . \mathrm{y}_{\mathrm{i}} \geq \mu$ which implies: $\lambda . \mathrm{B}+\lambda \cdot \mathrm{C} / \mathrm{n} \geq \mu$ and $\lambda . \mathrm{x}^{*}=\lambda . \mathrm{B}+\lambda . \mathrm{V}<$

$\mu$

and yields $: 1=\lambda . V<\lambda . C / n$ which means a contradiction. END.

Practical interpretation

We want now try to find some practical interpretation of the above considerations and thus ask the following question:

In which way does a variable change which increases the thickness of a polyedron while conserving the integrality constraint makes easier solving the related system of inequations?

\section{Remark}

The variable change associated with Hermite Normal decomposition doesn't always have this property.

In order to answer this question, we proceed by first writing the following descent heuristic, which work on any system $\mathrm{Ax} \leq \mathrm{b}$, which admits some integral solution and defines a bounded polyedron.

\section{Algorithm TEST-THICK}

Input : $\mathrm{A}, \mathrm{b}$ defining some polyedron $\mathrm{P}$ of $\mathrm{R}^{\mathrm{n}}$;

Output : $x$ such that $A x \leq b, x$ integral, or Failure ;

Initialize $\mathrm{x}$ in $\mathrm{Z}^{\mathrm{n}}$; Counter : $=0$;

While $\mathrm{x}$ not in $\mathrm{P}$ and Counter $\leq 100$ do

Randomly generate $\mathrm{p}$ in $\mathrm{N}^{\mathrm{m}}$; Possible ;

While not Possible do

Try to find $x^{\prime}$, which differs from $x$ by exactly one coordinate and such 


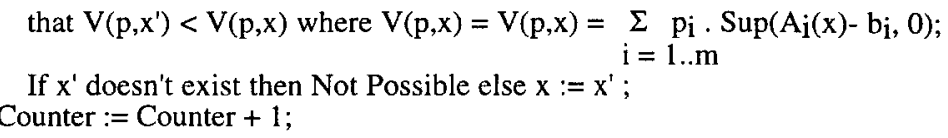

Then we compare, for a wide sample of such systems $A x \leq b$, the behaviour of this heuristic before and after some variable change $\mathrm{x}=\mathrm{UX}$, which induces the multiplication of the thickness of $\mathrm{P}$ by some coefficient $\alpha>1$. Then we get an evolution of the ratio:

$\mathrm{R}=$ Number of failures for TEST-THICK working on the initial system

Number ...on the system rewriten through the variable change $\mathrm{x}=\mathrm{UX}$

as follows:

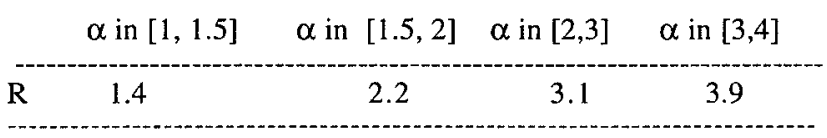

\section{Remark}

In order to systematically take profit from this kind of analysis, we shall need to be able to characterize (and compute) the variable change, which, for a given system $A x \leq b$, will make increase the thickness of the related polyedron. At this time, we can't answer this question.

\section{CONCLUSION}

We saw that a conveniently designed variable change may modify the arithmetical or geometrical characteristics of an ILP, and thus make easier its resolution. Still, several questions remain open. While a Hermite change of variable may speed the execution of some kind of cutting process, it may induce trouble with very large subdeterminants. On an other side, using an other kind of variable change in order to "smooth" the polyedron (to increase its thickness) may be usefull before applying some very simple descent processes, but it raises the question of finding a way to automatically determine such a well designed variable change.

\section{REFERENCES}

CHVATAL.V. (1985) Cutting planes and combinatorics. European Journal of combinatorics, 6, 217-26.

DAKIN.R.J. (1965) A tree search algorithm for mixed integer programming problems. The Computer Journal , 8, 250-255.

DAMICH.P.D, KANNAN.R and TROTTER.L. (1987) Hermite normal form computation using modulo determinant arithmetic. Math operat Research , 12.1, 50-59.

GAREY.M and JOHNSSON.D. (1979) Computer and intractability. W.Freeman and Co, N.Y.

GLOVER.F. (1966-7) "Generalized cuts in diophantine programming. Management Sciences, 13, 254-68.

GOMORY.R.E. (1963) An algorithm for integer solutions to linear programs, in Recent Advances in Math Programming, (R.L.Graves and P.Wolfe eds), Mac Graw Hill, N.Y, 269302.

LENSTRA.H. (1983) Integer Programming with a fixed number of variables. Maths of Operat Research , 8, 538-48.

SCHRIJVER.A. (1986) Theory of linear and integer programming. Wiley, Chichester.

SRINIVASAN.A.V. (1965) An investigation of some computational aspects of integer programming. $J A C M, 12,525-35$. 